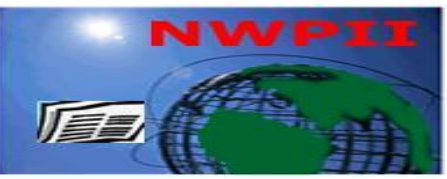

American Journal of Biomedical Sciences

ISSN: 1937-9080

nwpii.com/ajbms

\title{
Assessment and Comparison of the Skin Irritation Potential of Five Plant Extracts Using In Vitro and In Vivo Methods
}

\author{
Ning Su${ }^{1}$, Quan $\mathrm{An}^{2}$, Dan Zhao ${ }^{3}$, Hongyan Zheng ${ }^{1}$, Li Yang ${ }^{1}$, Xinshi Li ${ }^{1}$, Changtao Wang ${ }^{3,4 *}$ \\ ${ }^{1}$ Chinese Academy of Inspection and Quarantine, Daxing District, Beijing, China \\ ${ }^{2}$ Yunnan Baiyao Group Co., Ltd., Chenggong District, Kunming, China \\ ${ }^{3}$ Beijing Key Laboratory of Plant Resources Research and Development, Beijing Technology and Business University, \\ Haidian District, Beijing, China \\ ${ }^{4}$ Beijing Advanced Innovation Centre for Food Nutrition and Human Health, Beijing Technology and Business \\ University, Haidian District, Beijing, China. \\ Corresponding Author \\ Changtao Wang \\ School of Science \\ Beijing Technology and Business University \\ Beijing, China \\ Tel: 086-010-68984917 \\ Email: wangct@th.btbu.edu.cn
}

Received: 7 December 2016; | Revised: 27 February 2017; | Accepted: 6 March 2017

\begin{abstract}
The aim of this paper was to evaluate the skin irritant potential of five plant extracts for cosmetic use by in vitro and in vivo methods and to analyze the correlation between these different methods. Cell viability in fibroblasts and in the reconstructed human skin model was measured using the MTT assay to test cytotoxicity, and ELISA was used to detect IL-8 release. Finally, human skin patch tests were performed to validate the results obtained using the in vitro methods. The human patch results were compared with IL- 8 release results in fibroblasts and the MTT assay findings in Episkin ${ }^{\text {TM }}$. The MTT assay in fibroblasts showed that the five plant extracts exhibited low cytotoxicity. The human patch results showed that four of the five plant extracts showed weak irritant potential. The MTT assay results in Episkin ${ }^{\mathrm{TM}}$ were best correlated with the human patch test results with a Spearman's correlation coefficient of 0.6156. The Spearman's correlation coefficient between the human patch test and IL- 8 release results was 0.4617 . The MTT assay in Episkin ${ }^{\mathrm{TM}}$ was more sensitive than the IL- 8 release assay in fibroblasts to evaluate skin irritant potential. Our findings indicate that the five plant extracts are safe for cosmetic use.
\end{abstract}

Keywords: Skin irritation, Fibroblasts, Reconstructed human model, IL-8, Human skin patch test 


\section{Introduction}

Skin irritation testing is an important requisite of the preparation of pharmaceuticals and cosmetics. The most widely used method for assessing skin irritancy is the Draize test, which was developed in the 1940s and tests the harmfulness of chemicals to the human eye by dropping the test substance into one eye of a rabbit [1]. However, there are many drawbacks to this test, such as poor reproducibility, strong subjectivity, and the physiological dissimilarity between rabbit and human skin, which allows different responses to irritants [2-3]. In the past 40 years, as animal protection activity has arisen internationally, there is more awareness of the need to protect animal welfare [4]. In the past 20 years, to address ethical concerns of animal testing, the European Council has pursued a policy of reduction, refinement, and replacement (the 3Rs), promoting methods that avoid or replace the use of animals with alternative techniques [5].

Since 2013, cosmetic testing on animals was completely forbidden in the European Union and import and trade of products tested on animals are illegal acts. After decades of research into alternative testing methods, a series of mature methods are now used for the safety evaluation of cosmetics. Among these methods, EpiSkin and SkinEthic reconstituted human epidermis models have been validated by the European Union [6], so these alternative models could replace the Draize method that uses rabbits. In addition, bovine corneal opacity and permeability (BCOP), isolated chicken eye (ICE), and the hen's egg test on the chorioallantoic membrane (HET-CAM) are methods that are already certified by the Organization for Economic Cooperation and Development (OECD) and have been applied by some cosmetics companies [7]. Further, the above alternative methods that can replace animal testing are neither time-consuming nor expensive. In this paper, fibroblasts were used to build a simple and convenient method for evaluating the irritant potential of cosmetic ingredients.

Human skin fibroblasts can be surgically isolated from skin tissue, grown in vitro in culture medium, and are easily subcultured. Fibroblasts are widely used as a model to study the cellular physiology and biochemistry of skin and for clinical research. Fibroblasts play important roles in maintaining cell morphological and physiological characteristics, as well as in regulating the physiological function of skin. Research has shown that fibroblasts in derma can secrete various cytokines and extracellular matrix components, which function in regulating cell growth, proliferation, differentiation, and migration [8-9]. Additionally, fibroblasts can secrete inflammatory factors that influence skin irritation, allowing fibroblasts to be utilized in a highly sensitive assay to predict the potential for human skin irritation [10]. Thus, human skin cultures present several advantages, as these cells are closely relevant to organs and to species of concern, and are a mechanistically sound alternative to traditional skin irritation tests [11].

Assays using MTT (3-(4,5-dimethylthiazol-2yl)-2,5-diphenyltetrazolium bromide) to evaluate cytotoxicity and ELISA (enzyme-linked immunosorbent assay) to detect IL-1 $\alpha$, an inflammatory factor correlated with irritation, are frequently used for the prediction of skin irritation. In the present study, ELISA was used to detect IL-8 as a predictor of skin irritation potential. As one of the main inflammatory factors, increased IL-8 levels are detected in serum and body fluids during local inflammation. After stimulation by IL- $1 \alpha$, keratinocytes can express IL-8 [12]. Coquette et al. found that irritation induced SkinEthic models to release IL-8 [13]. The increased expression of IL-8 is closely related to tissue damage [14], which increases the concentration of intercellular $\mathrm{Ca}^{2+}$ and causes granule exocytosis [15]. Thus, the levels of IL-8 can be used to assess the degree of inflammatory disease. Bernhofer et al. detected elevated amounts of IL-8 in cultures containing fibroblasts and hypothesized that IL-8 might be secreted by fibroblasts after irritation or that fibroblasts may up-regulate the production of IL-8 by keratinocytes [16-17].

In this study, five plant extracts were tested as raw materials for cosmetic formulations were tested for the skin irritant potential. We tested the response at the cellular level in human fibroblasts using the MTT assay and an IL-8 release assay. Next, we used a reconstructed human skin model, and the MTT assay in Episkin ${ }^{\mathrm{TM}}$ was used to assess 
the sample irritant potential. Finally, the samples were tested using the human skin patch test to validate the irritant potential of the samples and then we analyzed the correlation between different methods.

\section{Experiment}

\subsection{Chemicals}

Camellia sinensis Kuntze extract ( Camellia ex.), Centella asiatica L. extract (Centella ex.), Rhodiola rosea L. extract (Rhodiola ex.), Sophora flavescens Aiton extract (Sophora ex.) and Phyllanthus emblica L. extract (Emblica ex.) were purchased from Beijing Merlin Technical \& Trading Company. According to the manufacturer, the extracts are considered stable for 24 months at room temperature. The five extracts were liquid samples and water-soluble.

The Camellia sinensis Kuntze extract is comprised of $30 \%$ tea polyphenols, $15.7 \%$ carbohydrate, $7.6 \%$ protein, and $46.7 \%$ water. The Centella asiatica L. Urban extract is comprised of $15 \%$ asiaticoside, $5 \%$ asiatic acid, $3 \%$ madecassic acid, $3.8 \%$ carbonhydrate, $2.1 \%$ protein, and $71.1 \%$ water. The Rhodiola rosea L. extract is comprised of $25 \%$ rhodioloside, $7.4 \%$ carbonhydrate, $2.8 \%$ protein, and $64.8 \%$ water. The Sophora flavescens Aiton extract is comprised of $8 \%$ matrine, $3 \%$ oxymatrine, $5 \%$ sophoranol, $12.3 \%$ carbonhydrate, $4.2 \%$ protein, and $67.5 \%$ water. The Phyllanthus emblica L. extract is comprised of $10 \%$, phyllemblic acid, $6 \%$ emblicol, $5 \%$ zeatin, $3.9 \%$ carbonhydrate, $1.5 \%$ protein, and $73.6 \%$ water.

Human skin fibroblasts were supplied by ScienCell Research Laboratories (Beijing, China). Episkin $^{\mathrm{TM}}$ was acquired from L'Oréal (Paris, France). Human IL-8 ELISA Kit was purchased from Beijing BLKW Biotechnology Co., Ltd.

\subsection{Cell culture}

Cells were removed from storage in liquid nitrogen and shocked to induce melting as quickly as possible in $37{ }^{\circ} \mathrm{C}$ water. The cells were then centrifuged for $5 \mathrm{~min}$ at $1000 \mathrm{rpm}$ and suspended in $1 \mathrm{ml}$ DMEM (Gibco, New York, USA) and transferred to T25 culture flasks (Corning, Shanghai, China). Approximately $5 \mathrm{ml}$ of DMEM was added and mixed uniformly before the cells were placed in a $5 \%(\mathrm{v} / \mathrm{v}) \mathrm{CO}_{2}$ incubator at $37{ }^{\circ} \mathrm{C}$. When the cell density reached $80 \%$ confluence, cells were washed twice with PBS (Corning, Shanghai, China). After digestion of the cell layer with $200 \mu \mathrm{l}$ of $0.25 \%$ trypsin (Gibco, New York, USA), cells were centrifuged at $1000 \mathrm{rpm}$ for 5 minutes. After removing the supernatant, $5 \mathrm{ml}$ DMEM in $10 \%$ FBS (Gibco, New York, USA) was added to resuspend the cells. Cell were then diluted to a density of $5 \times 10^{4}$ cells $/ \mathrm{ml}$ into the wells of a 96well tissue culture plate (BD Falcon, New York, USA) and were incubated for $24 \mathrm{~h}$ before performing cytotoxicity assays.

\subsection{In vitro model assays}

\subsubsection{MTT assay in fibroblasts}

Immediately after rinsing, the cells were evaluated for viability using the MTT assay, in which yellow MTT is reduced to the insoluble purple formazan by reductases located in the mitochondria of living cells. Cells treated with different concentrations (v/v) of test samples were incubated for $48 \mathrm{~h}$ before MTT testing. Cells without additional extract were used as a blank. Cells treated with SDS (Sigma-Aldrich Chemicals, St. Louis, USA) served as the positive control. A 5 $\mathrm{mg} / \mathrm{ml}$ stock solution of MTT (Sigma-Aldrich Chemicals, St. Louis, USA) was prepared in DMEM just prior to use and was warmed to $37{ }^{\circ} \mathrm{C}$ in a water bath. Cells were transferred to $96-$ well plates containing $20 \mu \mathrm{l}$ MTT medium $(5 \mathrm{mg} / \mathrm{mL})$ per well and placed in the humidified $5 \%(\mathrm{v} / \mathrm{v}) \mathrm{CO}_{2}$ incubator at $37^{\circ} \mathrm{C}$. After $4 \mathrm{~h}$ incubation, the nutrient solution was gently removed from the cells and 150 $\mu l$ DMSO (Gibco, New York, USA) was added to stop the MTT reaction. Cells were placed on the shaker for $5 \mathrm{~min}$ at slow speed to allow the reaction to complete. The sample absorbances were then measured at $570 \mathrm{~nm}$ using a spectrophotometer. The relative cell viability was calculated for each tissue as a percentage of the mean of the negative control tissues.

\subsubsection{IL-8 Assay}

To assess IL- 8 production, cells were seeded in 96 well plates at a concentration of $1 \times 10^{5}$ cells $/ \mathrm{ml}$. At $24 \mathrm{~h}$ post incubation, the culture medium was discarded and cell samples were treated with $0.01 \%$ 
(v/v) of each test extract. After incubation for $48 \mathrm{~h}$, the supernatant of the fibroblast culture medium was collected and stored at $-20{ }^{\circ} \mathrm{C}$. A specific highsensitivity ELISA was used to quantify the release of IL-8 in $500 \mu \mathrm{l}$ of fibroblast medium. The OD of the end products was measured at $450 \mathrm{~nm}$. The results were expressed in $\mathrm{pg} / \mathrm{mL}$.

\subsubsection{MTT assay in Episkin ${ }^{\mathrm{TM}}$}

The reconstructed human skin model (RhE) cells were equilibrated overnight at $37{ }^{\circ} \mathrm{C}$ and $5 \%$ $\mathrm{CO}_{2}$ in a humidified incubator. $10 \mu \mathrm{l}$ samples $(5 \%$ SDS as positive control and DPBS (Gibco, New York, USA) was used as the negative control) were applied to the apical side of the models and were exposed for 15 minutes. Next, the RhE were thoroughly rinsed with DPBS to complete exposure. The $\mathrm{RhE}$ were transferred to fresh medium and incubated for $42 \mathrm{~h}$. After incubation, tissues were transferred to 24-well plates containing $300 \mu \mathrm{l}$ MTT medium $(1 \mathrm{mg} / \mathrm{mL})$ per well and placed in the $37{ }^{\circ} \mathrm{C}, 5 \% \mathrm{CO}_{2}$ humidified incubator for $3 \mathrm{~h}$. The tissues were then removed from the MTT medium. The formazan salt was extracted from the tissues by transferring them to 24-well plates containing $2 \mathrm{~mL}$ of isopropanol (Sigma-Aldrich Chemicals, St. Louis, USA). The submerged tissues were incubated overnight at room temperature and protected from light. After the overnight extraction, the plates were shaken for approximately $15 \mathrm{~min}$. The optical density of the extracted formazan was determined by transferring $200 \mu \mathrm{l}$ of each extraction solution into a new, optically clear 96-well plate. Isopropanol was used as a blank. The plates were read using a spectrophotometer at $570 \mathrm{~nm}$. The relative cell viability was calculated for each tissue as the percent of the mean of the negative control tissues.

\subsection{In vivo irritation testing}

\subsubsection{Human skin patch testing}

Thirty volunteers between 20 and 35 years of age, who were not suspected of having any allergic contact dermatitis, were subjected to testing. The 5 plant extracts $(5 \% \mathrm{v} / \mathrm{v})$ were applied with Haye's test chambers (HAIJE SERVICE B.V., Washington, USA) to a $70 \mathrm{~cm}^{2}$ area of skin on each volunteer's back. The test materials were removed after $48 \mathrm{~h}$ and the treated sites were examined after $30 \mathrm{~min}$, $24 \mathrm{~h}$, and $48 \mathrm{~h}$. Five types of reactions were classified according to the International Contact Dermatitis Research Group (ICDRG) scoring system [18]. The five reactions include negative reaction $(-)$, possible reaction $( \pm$, faint erythema only), weak positive reaction $(+$, erythema, infiltration, and possible papules), strong positive reaction $(++$, erythema, infiltration, papules or vesicles), and extreme positive reaction (+++, intense erythema, infiltration, and coalescing vesicles). The human skin patch test score was calculated by the following equation.

Mean score $=($ Grade $\times$ No. of responders $) /(4$ $($ maximum grade $) \times 30) \times 100 \times 1 / 2$

\subsection{Statistical analyses}

All experiments were performed at least three times. The statistical analyses were carried out using GraphPad Prism 5 (GraphPad Software, Inc., San Diego, USA) including analysis of median inhibitory concentration $\left(\mathrm{IC}_{50}\right)$ and Spearman's rank correlation.

\section{Results}

\subsection{MTT assay in fibroblasts}

Fibroblasts were exposed to test extracts or controls for $48 \mathrm{~h}$ before the MTT assay. As seen in Figure 1, as the concentration of samples increased to $10 \%(\mathrm{~V} / \mathrm{V})$, the five extracts significantly reduced cell viability. For Camellia ex. and Rhodiola ex., when the sample concentration was lower than $1 \%$, the fibroblast viability was higher than $100 \%$. The calculated $\mathrm{IC}_{50}$ values of the plant extracts in the fibroblast MTT assay are shown in Table 1. The order of cytotoxicity of the samples, from strong to weak, was Emblica ex., Centella ex., Sophora ex., Rhodiola ex., and Camellia ex..

\subsection{IL-8 release}

The extracts exhibited no cytotoxicity at $0.01 \%$ concentration. As cytotoxicity affects the release of IL-8, cells were cultured with $0.01 \%$ test extracts for $2 \mathrm{~h}$ as shown in Figure 2. There was less IL-8 release than in the negative control after treatment with $0.01 \%$ Camellia ex, Rhodiola ex, Emblica ex., Centella ex., or Sophora ex. 


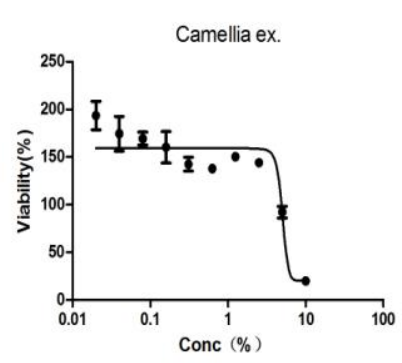

(a)



(c)



(e)

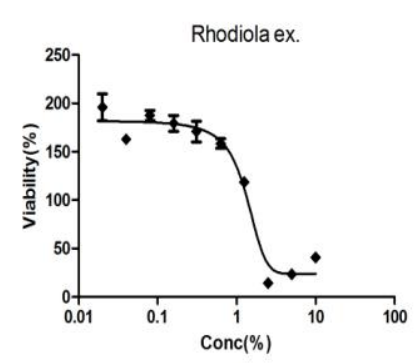

(b)

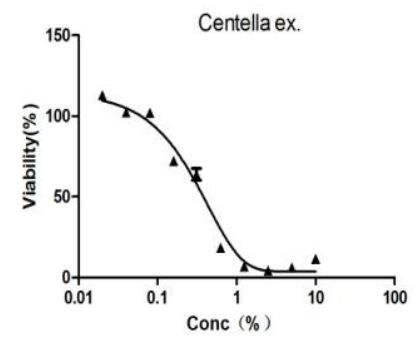

(d)



(f)
Figure 1. Cellular viability measured by MTT assay after treatment of cells with the following substances

Table 1. $\mathrm{IC}_{50}$ of the plant extracts in the fibroblast MTT assay

\begin{tabular}{cc}
\hline Sample name & $\mathrm{IC}_{50}$ \\
\hline Camellia ex. & $2.828 \%$ \\
Rhodiola ex. & $1.156 \%$ \\
Emblica ex. & $0.037 \%$ \\
Centella ex. & $0.049 \%$ \\
Sophora ex.. & $0.098 \%$ \\
SDS & $0.540 \mu \mathrm{g} / \mathrm{ml}$ \\
\hline
\end{tabular}

Am. J. Biomed. Sci. 2017, 9(1), 20-28; doi: 10.5099/aj170100020

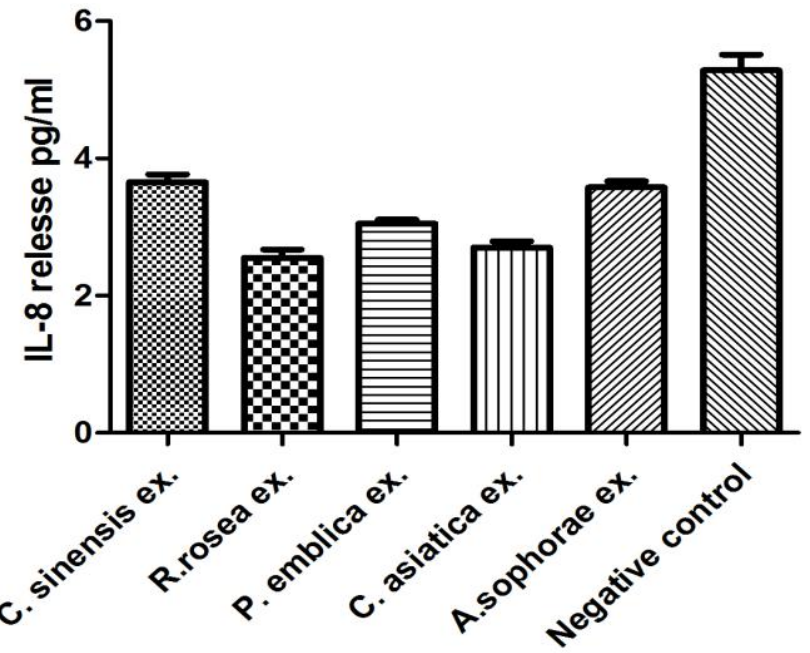

Figure 2. ELISA quantification of IL-8 released into the culture medium after $2 \mathrm{~h}$ exposure to the following substances.

\subsection{MTT assay in Episkin ${ }^{\mathrm{TM}}$}

The relative cell viability was calculated for each $\mathrm{RhE}$ as the percentage of the mean of the RhE negative control. For both models, if the percentage of viability was $>50 \%$, the substance was classified as non-irritant (EU classification: no label; GHS classification: no category). If the percentage of viability was $\leq 50 \%$, the substance was predicted as irritant (EU classification: R38, R41 or R36, depending on the model; GSH classification: Category 1 or Category 2). Treatment with all samples resulted in viability percentages higher than 50\% (Figure 3), indicating that the five samples were non-irritant.



Figure 3. Viability of the Episkin ${ }^{\mathrm{TM}}$ after treatment with the five plant extracts for 15 minutes. 5\% SDS was used as positive control. 


\subsection{Human skin patch test results}

The human skin patch test results are shown in Table 2. Among the five plant extracts, Camellia ex. appeared to be a stronger irritant than the other test extracts, as it induced a weak positive reaction in one subject and two possible reactions in two subjects. Rhodiola ex. appeared to be the least irritating of the extracts, as it caused neither possible reactions nor positive reactions. Emblica ex., Centella ex., and Sophora ex. induced two, four, and one possible reactions, respectively.

\subsection{Statistical analysis}

Spearman's rank correlation coefficient was calculated to analyze the correlation between in vitro and in vivo irritation tests. Results showed that the results for the human skin patch test and viability of Episkin ${ }^{\mathrm{TM}}$ were well correlated (Rs=0.6156). The Spearman's correlation coefficient between the human patch test and IL-8 release results was 0.4617 . The results were shown in Figure 4.

Table 2. Human skin patch test results after treatment with the 5 test extracts

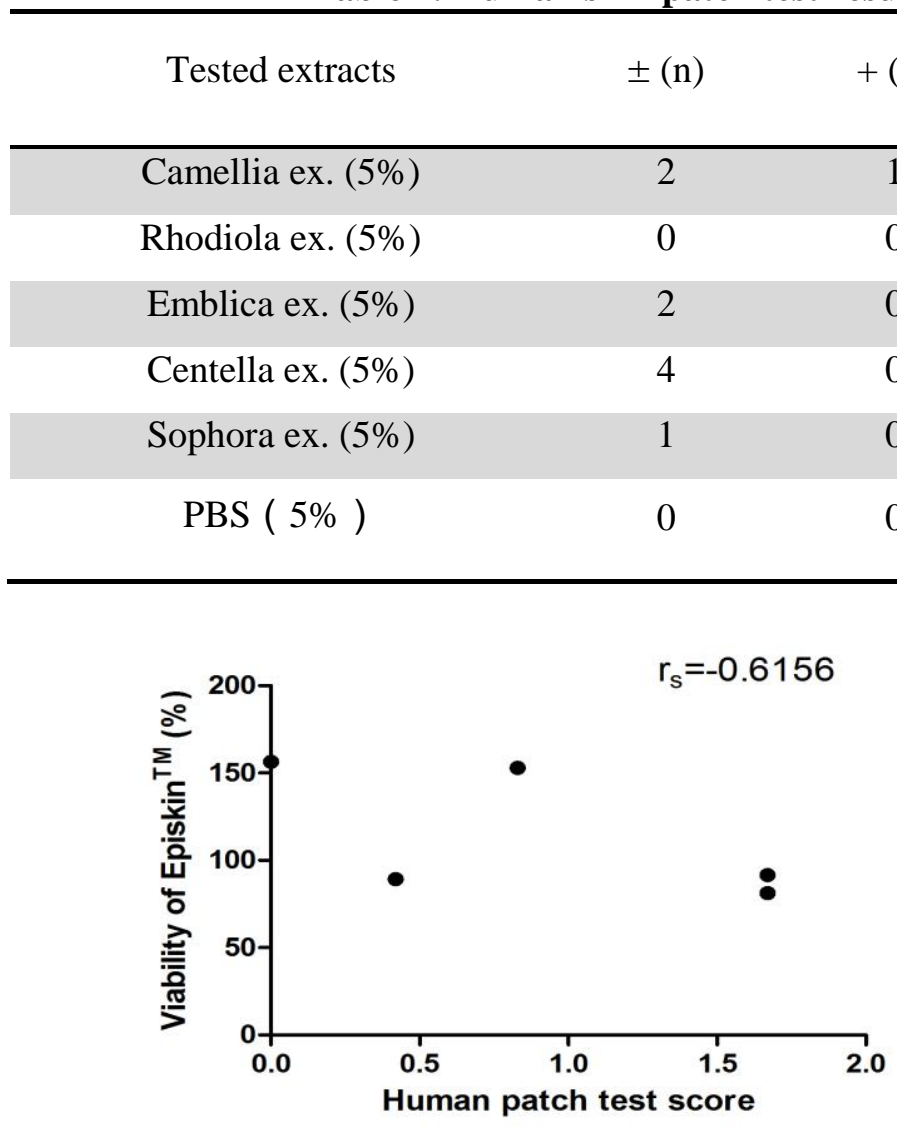

(a)



(b)

Figure 4. (a) Correlation between human patch test score and viability of Episkin ${ }^{\mathrm{TM}}$. (b) Correlation between human patch test score and IL-8 relative release from cells.

\section{Discussion}

In vitro skin irritation tests were originally developed to predict the human skin irritation potential of raw chemicals and cosmetic products. These tests have also been used to screen medical device materials, components, and other products for potential skin irritation. Here, we reported results of a three-component study designed to evaluate the irritant potential of five different plant extracts. This study included MTT assay and inflammatory response as indicated by IL-8 release in cultured human fibroblasts, MTT assay in RhE, and the human skin patch test. Irritation is defined as the production of reversible damage to the skin following the application of a test substance for up to $4 \mathrm{~h}$ [19-20]. The more significant the initial 
injury, including cell death, the stronger the irritant. The MTT assay was used to evaluate cell viability. The release of IL- 8 was measured because initial experiments revealed that IL-8 was released after the treatment of cells with cosmetics or extracts of raw materials. In the initial stage of skin inflammation, IL-8 release may lead to the migration of dendritic cells, which causes monocyte and neutrophil recruitment [21].

The main object of our investigation was to use an in vitro cell culture model to predict the irritant potential of 5 cosmetic ingredient extracts. After cells were exposed to each extract for $48 \mathrm{~h}$, the cellular viability was assayed. The results enabled us to differentiate extracts with no cytotoxicity from products with moderate cytotoxicity at the cellular level. Our results indicated that the whole plant extracts tested here do not generate serious injury to fibroblasts. Cell viability was greater than $50 \%$ after treatment with the individual plant extracts at $0.03 \%$ for $48 \mathrm{~h}$. According to the IL-8 release results, Camellia ex. acted as a stronger irritant than the other extracts and Rhodiola ex. was the weakest irritant. This result was not well correlated with the human skin patch test results $(\mathrm{Rs}=0.4617)$. The release of IL-8 was previously shown to correlate with high irritant potential and severe cytotoxicity, and we also observed IL-8 release upon exposure to the test extracts [22-23]. The degree of IL-8 release induced by the five plant extracts was lower than that of the negative control, which may partially explain the human skin patch test results. IL- $1 \alpha$ plays an important role in skin irritation, and the up-regulation of IL- $1 \alpha$ may induce IL-8 release. Protein kinase C (PKC) could regulate the activation of a nuclear factor via a responsive enhancer in the 5'-flanking region of the IL-8 gene to promote IL-8 synthesis [24-26]. The amount of IL-8 release is determined by many factors that affect the synthesis of IL- $1 \alpha$ and the activity of nuclear factor(s), and further experiments are necessary to explore the multiple factors that influence IL- 8 synthesis in this system.

The five cosmetic ingredient test extracts showed no obvious effect to inhibit the viability of Episkin $^{\mathrm{TM}}$. Rhodiola ex. and Emblica ex. greatly increased the viability of Episkin ${ }^{\mathrm{TM}}$. Camellia ex., Centella ex., and Sophora ex. weakly inhibited the viability of Episkin ${ }^{\mathrm{TM}}$. These results agreed with the results of the human skin patch test (Rs=0.6156).

In general, tests using in vitro cell cultures and the reconstructed human skin model are more efficient and less costly than human skin irritation tests, and reduce the use of human and animal skin [27]. However, there are limitations to the applicability of human skin culture methods, as only a few major skin cell types (e.g. epidermal keratinocytes, dermal fibroblasts, or both in coculture) are typically used [25].

In vitro cytotoxicity has often been used to fairly accurately predict skin irritation potential. There is close agreement between human skin patch test results and the results from the MTT assay in Episkin $^{\mathrm{TM}}$. However, disagreement between in vitro cytotoxicity data and the skin patch test results were observed in the present study using plant extracts. Using human skin fibroblasts, we found that the $0.01 \%$ plant extracts exhibited no cytotoxicity. The IL-8 ELISA data indicated that the whole five plant extracts induced lower IL-8 release than the control, which indicated the potential safety of the extracts. However, the IL-8 ELISA results were not well related to the results of the human skin patch test. Because human skin is made up of a variety of cells and a complex organizational structure that includes keratinocytes, langerhans cell, melanocytes, and other cells, fibroblasts alone are unable to perfectly mimic human skin. The Episkin ${ }^{\mathrm{TM}}$ results were in better agreement with the in vivo results, indicating that the five extracts were not severely irritating to human skin. Further experiments are necessary, with special attention required to explore the influence of other factors on IL-8 secretion. In the present study, a direct correlation was found between Episkin ${ }^{\mathrm{TM}}$ viability and skin irritation results as determined by human skin patch tests (Rs=-0.6156).

\section{Acknowledgments}

This research was supported by the Quality Inspection for Public Welfare of Special Scientific Research funded projects (201310132) and Quality Inspection for Public Welfare of Special Scientific Research funded projects (201410019) from China. 


\section{References}

1. Draize, J. H.; Woodward, G.; Clavery, H. O. Methods for the study of irritation and toxicity of substances applied topically to the skin and mucous membranes, Journal of Pharmacology and Experimental Therapeutics,1944, 82(3), 377-390.

2. Marzulli, F. N.; Maibach, H. I. The rabbit as a model for evaluating skin irritants: a comparison of results obtained on animals and man using repeat skin exposures, Food and Cosmetics Toxicology, 1975, 13(5), 533-540. DOI: 10.1016/0015-6264(75)90008-5

3. Scott, R. C.; Corrigan, M. A.; Smith, F.; Mason, H. The influence of skin structure on permeability: an intersite and interspecies comparison with hydrophilic penetrants, Journal of Investigative Dermatology,1991, 96(6), 921-925. DOI: 10.1111/15231747.ep12475447

4. Casas, J. W.; Lewerenz, G. M.; Rankin, E. A.; Willoughby Sr., J. A.; Blakeman, L. C.; McKim Jr., J. M.; Coleman, K. P. In vitro human skin irritation test for evaluation of medical device extracts, Toxicology in Vitro, 2013; 27(8), 2175-2183. DOI: $10.1016 / j . t i v .2013 .08 .006$

5. Singh, J. The National Centre for the Replacement, Refinement, and Reduction of Animals in Research, Journal of Pharmacology \& Pharmacotherapeutics, 2012, 3(1), 87-89.

6. ESAC, Statement on the Scientific Validity of in vitro tests for Skin Irritation Testing, 2008.

7. Adriaens, E.; Barroso, J.; Eskes, C.; Hoffmann, S.; McNamee, P.; Alépée, N.; Bessou-Touya, S.; De Smedt, A.; De Wever, B.; Pfannenbecker, U.; Tailhardat, M.; Zuang, V. Retrospective analysis of the Draize test for serious eye damage/eye irritation: importance of understanding the in vivo endpoints under UN GHS/EU CLP for the development and evaluation of in vitro test methods, Archives of Toxicolog, 2014, 88(3), 701-723. DOI: 10.1007/s00204-013-1156-8

8. Bi, D.; Chen, F. G.; Zhang, W. J.; Zhou, G. D.; Cui, L.; Liu, W.; Cao, Y. Differentiation of human multipotent dermal fibroblasts into isletlike cell clusters, BMC Cell Biology, 2010,11:46. DOI:10.1186/1471-2121-11-46
(2010).

9. Mostafa, T.M.; Ibrahim, H.; Badra, EK; ElBerri, EK. Role of commiphora Mol Mol and doxycycline in prophylaxis of spontaneous bacterial peritonitis in Egyptians cirrhotic patients. Am. J. Biomed. Sci. 2015, 7(3), 176189 . DOI: $10.5099 / a j 150300176$

10. Moulin, V. J.; Reconstitution of skin fibrosis development using a tissue engineering approach, Methods in Molecular Biology, 2013;961, 287-303. DOI: 10.1186/1471-212111-46

11. Lee, J. K.; Kim, D. B.; Kim, J. I.; Kim, P. Y. In vitro cytotoxicity tests on cultured human skin fibroblasts to predict skin irritation potential of surfactants, Toxicology. in Vitro,2000, 14(4), 345-349. DOI: 10.1016/S0887-2333(00)00028$\underline{X}$

12. Wilmer, J. L.; Luster, M. I. Chemical induction of interleukin-8, a proinflammatory chemokine, in human epidermal keratinocyte cultures and its relation to cytogenetic toxicity, Cell Biology, Toxicology, 1995, 11(1), 37-50. DOI: 10.1007/BF00769991

13. Coquettea, A.; Berna, N.; Vandenbosch, A.; Rosdy, M.; De Wever, B.; Poumay, Y.; Analysis of interleukin-1 $\alpha$ (IL-1 $\alpha$ ) and interleukin-8 (IL-8) expression and release in in vitro reconstructed human epidermis for the prediction of in vivo skin irritation and/or sensitization, Toxicology in Vitro, 2003, 17(3), 311-321. DOI: 10.1016/S08872333(03)00019-5

14. Mohammadzadeh, M.; Mueller, M.; Hultsch, T.; Enk, A.; Saloga, J.; Knop, J. Enhanced expression of IL-8 in normal keratinocytes and normal human keratinocytes cell line $\mathrm{HaCaT}$ in vitro after stimulation with contact sensitizers, tolerogens and irritants, Experimental. Dermatology, 1994, 3(6), 298-303. DOI: $\quad 10.1111 / j .1600-$ 0625.1994.tb00292.x

15. Barker, J. N. W.; Mitra, R. S. C.; Griffith, E. M.; Bixit, V. M.; Nickoloff, B. J. Keratinocytes as initiators of inflammation, Lancet, 1991,337(8735), 211-214. DOI: 10.1016/0140-6736(91)92168-2

16. Boxman, I. L. A.; Ruwhof, C.; Boerman, O. C.; Löwik, C. W.; Ponec; M. Role of fibroblasts in the regulation of proinflammatory interleukin IL-1, IL6 and IL-8 levels induced by keratinocyte-derived IL-1, Archives of Dermatological Reserch.,1996,288(7), 391-398. DOI: 10.1007/BF02507108

17. Bernhofer, L. P.; Seiberg, M.; Martin, K. M. The influence of the response of skin equivalent systems 
to topically applied consumer products by epithelial mesenchymal interaction, Toxicology In Vitro, 1999, 13(2), 219-229. DOI: 10.1016/S08872333(98)00087-3

18. Wahlberg, J. E. "Patch Testing," in Text book of Contact Dermatitis, SpringerVerlag, 2001, pp. 241268.

19. OECD, OECD Test Guidelines for the Testing of Chemicals, Section 4, Test No. 404: acute dermal irritation/corrosion Paris, France: OECD DOI:10.1787/9789264070622-en,(2002).

20. UNECE, Skin corrosion/irritation, globally harmonized system of classification and labelling of chemicals (GHS) $4^{\text {th }}$ Ed. United Nations Economic Commission for Europe, New York and Geneva, (2011) pp.121-131.

21. Henkelsa, K. M.; Frondorfa, K.; GonzalezMejiab, M. E. IL-8-induced neutrophil chemotaxis is mediated by Janus kinase 3 (JAK3), FEBS Letters., 2011;585(1), 159-166. DOI: 10.1016/j.febslet.2010.11.031

22. Melonia, M.; Pauly, A.; De Servi, B.; Le Varleta, B.; Baudouin, C.; Occludin gene expression as an early in vitro sign for mild eye irritation assessment, Toxicology in Vitro, 2010; 24(1), 276-285. DOI: 10.1016/j.tiv.2009.08.016

23. Debbasch, C.; Ebenhahn, C.; Dami, N.; Pericoi, M.; Van den Berghe, C.; Cottin, M.; Nohynek, G. J. Eye irritation of low-irritant cosmetic formulations: correlation of in vitro results with clinical data and product composition, Food and Chemical Toxicology, 2005;43(1), 155165. DOI: $10.1016 /$ j.fct.2004.09.004

24. Brat, D. J.; Bellail, A. C.; Van Meir, E. G. The role of interleukin-8 and its receptors in gliomagenes is and tumoral angiogenesis, Neuro-Oncology, 2005;7(2), 122-133. DOI: 10.1215/S1152851704001061

25. Chabot-Fletcher, M.; Breton, J.; Lee, J.; Young, P.; Griswold, D.E. Interleukin-8 production is regulated by protein kinase $\mathrm{C}$ inhuman keratinocytes, The Journal of Investigative Dermatology, 1994;103(4), 509515. DOI: 10.1111/1523-1747.ep12395658

26. Lee, S. J.; Cho, S. A.; An, S. S.; Na, Y.-J.; Park, N.-H.; Kim, H.-S.; Lee, C.-W.; Kim, H.K.; Kim, E.-K.; Jang, Y.-P.; Kim, J.-W. Alstonia scholaris $\mathrm{R}$. Br. significantly inhibits retinoid-induced skin irritation in vitro and in vivo, Eviddence-Based Complementary and Alternative medicine. DOI: $10.1155 / 2012 / 190370$

27. Hirotaa, M; Kouzuki, H.; Ashikaga, T.; Sono, S.; Tsujita, K.; Sasa, H.; Aiba, S. Artificial neural network analysis of data from multiple in vitro assays forprediction of skin sensitization potency of chemicals, Toxicology in Vitro,2013, 27(4), 1233-1246. DOI: 10.1016/j.tiv.2013.02.013 